\title{
Progressive Multifocal Leukoencephalopathy and Systemic Lupus Erythematosus: Focus on Etiology
}

\author{
Shala Ghaderi Berntsson ${ }^{a, b} \quad$ Evangelos Katsarogiannis ${ }^{a, b}$ \\ Filipa Lourenço ${ }^{c}$ Maria Francisca Moraes-Fontes ${ }^{c}$ \\ Departments of ${ }^{a}$ Neuroscience and ${ }^{b}$ Neurology, Uppsala University Hospital, Uppsala,

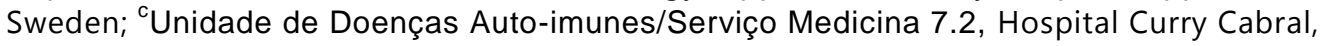 \\ Centro Hospitalar Lisboa Central, Lisbon, Portugal
}

\section{Key Words}

Progressive multifocal leukoencephalopathy · Systemic lupus erythematosus · Lymphopenia

\begin{abstract}
Progressive multifocal leukoencephalopathy (PML) caused by reactivation of the JC virus (JCV), a human polyomavirus, occurs in autoimmune disorders, most frequently in systemic lupus erythematosus (SLE). We describe a HIV-negative 34-year-old female with SLE who had been treated with immunosuppressant therapy (IST; steroids and azathioprine) since 2004. In 2011, she developed decreased sensation and weakness of the right hand, followed by vertigo and gait instability. The diagnosis of PML was made on the basis of brain MRI findings (posterior fossa lesions) and JCV isolation from the cerebrospinal fluid (700 copies $/ \mathrm{ml})$. IST was immediately discontinued. Cidofovir, mirtazapine, mefloquine and cycles of cytarabine were sequentially added, but there was progressive deterioration with a fatal outcome 1 year after disease onset. This report discusses current therapeutic choices for PML and the importance of early infection screening when SLE patients present with neurological symptoms. In the light of recent reports of PML in SLE patients treated with rituximab or belimumab, we highlight that other IST may just as well be implicated. We conclude that severe lymphopenia was most likely responsible for JCV reactivation in this patient and discuss how effective management of lymphopenia in SLE and PML therapy remains an unmet need.
\end{abstract}




\section{Case Reports in Neurology}

Ghaderi Berntsson et al.: Progressive Multifocal Leukoencephalopathy and Systemic Lupus Erythematosus: Focus on Etiology

\section{Introduction}

Progressive multifocal leukoencephalopathy (PML) is a rare and devastating demyelinating disease of the central nervous system. The disease is caused by reactivation of a polyomavirus, the JC virus (JCV), that was first described as a rare complication of chemotherapy in patients with lymphoproliferative disorders [1]. The dramatic increase of PML incidence during the AIDS epidemic in the 1980s and the occurrence of PML in patients with idiopathic CD4+ lymphopenia [2] pointed to the importance of CD4+ T cells in the immune control of JCV infections. Asymptomatic primary JCV infection actually occurs in up to $80 \%$ of the individuals, the virus remaining latent in lymphocytes and glial cells. Both T- and Bcell effector functions are required to prevent viral reactivation [3]. In autoimmune disorders, PML occurs mostly in patients with systemic lupus erythematosus (SLE) [4]. Of note, most SLE patients who develop PML have been either subjected or are concomitantly under immunosuppressant therapy (IST), with a diverse combination of therapeutic regimens that causes generalized immunosuppression. Steroids, azathioprine, mycophenolate mofetil, cyclophosphamide, methotrexate, thalidomide, dapsone and other specifically targeted therapies, such as rituximab or belimumab, are the treatments usually prescribed [5]. While the main cause of PML in SLE patients is likely to be therapy-related immunodeficiency, there is no correlation between the intensity of IST and PML development in SLE patients, suggesting that alternative mechanisms of JCV reactivation may exist in some patients [6]. Most case reports on SLE patients with PML lack data on total lymphocytes or lymphocyte subsets despite the fact that when reported, CD4+ T-cell counts are severely reduced [7]. Here, we report on a patient with SLE and persistent lymphopenia who developed PML.

\section{Case Presentation}

A young HIV-negative Portuguese female was diagnosed with SLE at the age of 12 on the basis of polyarthralgias, pneumonitis, high fever and autoantibody reactivities including antinuclear antibody (ANA) and anti-double-stranded (ds) DNA. Additional cumulative features relating to SLE consisted of photosensitivity, Jaccoud arthropathy, alopecia and fatigue, and ongoing positivity for anti-Smith-/ribonucleoprotein, antinucleosome and antihistone, quantified at 3+, with persistent absence of anti-SSA and anti-SSB (Euroimmun). Case records show that in 2004, for a period of 7 months, the anti-dsDNA antibody titer fluctuated between 1/10 and 1/30 (positive values by Crithidia luciliae immunofluorescence assay), after which time the test became negative. Thereafter, her illness was characterized by intermittent arthralgia of the small joints of the hands, knees and ankles. There was persistent hypocomplementemia and polyclonal hypergammaglobulinemia, with a high IgG (peak value 2,390 mg/dl; upper limit of normal 1,400), normal IgM and IgA concentrations. From 2004 to 2011, her white blood and total lymphocyte counts ranged from 2,300 to $3,900 \times 106 / 1$ and from 600 to $1,400 \times 106 /$, respectively, with mean counts of 3,000 and 1,000 $\times 106 / \mathrm{l}$, respectively. Lymphocyte phenotyping by flow cytometry was not performed during this time. The hemoglobin concentration and platelet counts were normal. She was not taking hydroxychloroquine and had been started on azathioprine $50 \mathrm{mg} /$ day and deflazacort 6 $\mathrm{mg} /$ day, which were continued from 2004 to February 2012. At her last regular follow-up visit in December 2011 she complained of decreased sensation and weakness of the right hand. An electromyography was planned. There were no clinical signs of lupus disease activity with an ANA titer of $1 / 320$, negative anti-dsDNA, normal C3 $(95.4 \mathrm{mg} / \mathrm{dl})$, slightly reduced C4 of $8.25 \mathrm{mg} / \mathrm{dl}$ (normal values 10-34) and normal urinary sediment. 
Ghaderi Berntsson et al.: Progressive Multifocal Leukoencephalopathy and Systemic Lupus Erythematosus: Focus on Etiology

In February 2012, at the age of 34, she complained of nausea, vertigo, limb ataxia and gait instability. At first, only slight ataxia of the right arm was noted. She was admitted to hospital and while waiting for a cerebral MRI, her symptoms rapidly deteriorated with episodes of vomiting and worsened limb ataxia, which became bilateral. Gadolinium-enhanced MRI of the brain revealed patchy high-signal intensity lesions on T2-weighted images in the right cerebellar peduncle without diffusion restriction (fig. 1). MRI angiography and a conventional cerebral angiogram showed no abnormalities of the vascular system and no signs of vasculitis. The total lymphocyte count was $800 \times 106 / \mathrm{l}$; there was no anemia or evidence of renal or hepatic dysfunction, and ANA and anti-dsDNA were positive, with a low concentration of complement components C3 and C4. Blood serology was negative for herpes simplex virus, cytomegalovirus, Epstein-Barr virus, enterovirus, varicella-zoster virus, human Tlymphotropic virus types 1 and 2, HIV, lyme disease and syphilis. Cerebrospinal fluid examination (CSF) revealed: (1) normal cell counts, protein, glucose, lactate, and immunoglobulin index, no oligoclonal bands and absent borrelia antibodies; (2) negative Gram stain, negative cultures for bacteria and negative microbiologic PCR for mycobacteria, herpes simplex virus, varicella-zoster virus, cytomegalovirus, Epstein-Barr virus, human T-lymphotropic virus types 1 and 2, Cryptococcus spp. and other fungi; (3) negative angiotensin-converting enzyme and glial fibrillary acidic protein; (4) negative paraneoplastic and inflammatory antibody screen (anti-Hu, Yo, Ri and Purkinje cells, NMDA receptor, MA-2), MBP, MOG and neuromyelitis optica, and (5) slight increase in polygonal IgG and neurofilament. CSF PCR was positive for JCV (700 copies/ml), which, together with the clinical data and MRI findings, clinched the diagnosis of PML.

Steroids and azathioprine were discontinued. Treatment with cidofovir $5 \mathrm{mg} / \mathrm{kg}$ was started, the first 3 treatments 4 days apart and then every second week, to which mirtazapine $45 \mathrm{mg}$ daily was added. Despite these therapeutic measures, there was clinical deterioration with abnormal saccadic eye movements and failure of fixation, scanning dysarthria and more prominent gait ataxia, requiring gait assistance to prevent falls. Two weeks later, a new MRI showed progress of signal abnormality in the cerebellum and further extension now involving the brain stem and thalami. After 2 months, mirtazapine was reduced to 30 $\mathrm{mg}$ /day to avoid excess sedation, and mefloquine $250 \mathrm{mg} /$ day once a week was started. Clinical deterioration as well as progression of MRI signal intensity in the pons and periventricular demyelination continued at 3 months after the onset of her symptoms. The patient experienced additional paresthesia on the left side of her face and swallowing difficulties. Cytarabine was added to cidofovir based on previously reported successful treatment results [8].

Thereafter, her clinical state deteriorated with progressive neurological dysfunction, lethargy and confusion. The patient was bedridden and no longer able to communicate due to severe dysarthria. She was suffering from constant nausea, vomiting, diplopia, nystagmus, dysphagia, hemiparesthesia, right-sided hemiparesis and severe limb, gait and truncal ataxia. She also developed severe depression with mood changes, crying outbursts, despair, insomnia and loss of appetite. At 5 months after hospital admission, the MRI showed further disease progression in the pons (fig. 2). During treatment, CD4+ levels and the CD4/CD8 ratio ranged between 262 and $381 \times 106 / 1$ and 0.7 and $1.1 \times 106 /$ l, respectively. A slight initial decrease in JCV copies in the CSF was followed by a further increase to 1,400 and 2 months later to 2,500 copies/ml, after which there was a continuous increase up to $3,900 \mathrm{copies} / \mathrm{ml}$, the latter measured at 6 months after hospital admission. She was then transferred to Portugal where treatment with cidofovir and cytarabine were discontinued as she suffered serious recurrent infections. At 12 months after the initial diagnosis, a new MRI showed bilateral periventricular high-signal intensity lesions (fig. 3), and the patient died in a persistent vegetative state 1 year after the start of her disease. 
Ghaderi Berntsson et al.: Progressive Multifocal Leukoencephalopathy and Systemic Lupus Erythematosus: Focus on Etiology

\section{Discussion}

SLE is an autoimmune disease of unknown etiology with heterogeneous manifestations involving several organ systems, including the brain. Patients with neuropsychiatric lupus may present a wide spectrum of syndromes such as headache, seizures, aseptic meningitis, stroke, psychosis, cranial neuropathy, demyelinating syndromes, movement and cognitive disorders [9]. Medication side effects, metabolic abnormalities and particularly infections can be a major cause of SLE central nervous system syndromes [9] and were therefore investigated promptly in the diagnostic workup. Our patient fulfilled the current diagnostic criteria for PML, consisting of neurological features, typical imaging and detection of JCV in the CSF by PCR [10].

Neurological features vary widely in PML patients. Visual disturbances, motor weakness, altered mental state and sensory deficit remain the most common ones, whereas symptoms due to mass effect or epilepsy are quite rare [11]. MRI is the most sensitive imaging method for the investigation of suspected PML, typical lesions appearing hyperintense on fluid-attenuated inversion recovery and T2-weighted sequences [3]. In our patient, initial suspicion of peripheral nerve entrapment delayed the diagnostic workup, as a consequence of which therapy was instituted at a late stage, the latter probably contributing to refractoriness to therapy, with a relentless clinical deterioration reflected by increasing MRI lesions, leading to death.

Increased susceptibility to severe bacterial infections has been described in association with lymphopenia defined by $<1,000$ lymphocytes $\times 106 / 1$ [12]. Lymphopenia in SLE is usually regarded as a disease manifestation, targeting lymphocytes or their precursors as an expected side effect of IST. However, interestingly, lymphopenia has been observed in up to $70 \%$ of the untreated patients at disease onset, suggesting that it may be a promoter rather than a consequence of the SLE disease process [13]. There are no current recommendations for the investigation and treatment of SLE patients with severe or persistent lymphopenia, in the absence or presence of IST. As a result, lymphopenia is frequently ignored in clinical practice when we believe that instead it should constitute a major clinical concern. Current recommendations for dealing with lymphopenia are poor. These essentially support maintaining a lymphocyte count $>1,000$ lymphocytes $\times 106 /$ l, exclusion of infections and lymphoproliferative disease, and principally advocate adjustment of IST without proposing replacement therapies [14]. Our patient presented with very low CD4+ T-cell counts and a low CD4/CD8 T-cell ratio, which most likely contributed to the JCV reactivation.

Treatment of SLE patients with PML should eliminate the virus and increase lymphocyte counts without aggravating any SLE manifestations. Highly active antiretroviral therapy has reduced mortality rates in HIV-positive patients with PML [3]. However, the treatment of non-HIV PML remains a challenge. Classical options have been based on immunomodulation and antiviral therapy. Cidofovir, an antiviral nucleotide analogue, showed some efficacy in the treatment of HIV-positive patients with PML but it has been unsuccessful in HIV-negative patients [2]. Cytarabine, a chemotherapy agent (nucleoside arabinoside), has been one of the most frequently used drugs despite commonly encountered adverse effects of nausea and bone marrow toxicity, but its use remains controversial. A randomized controlled trial of cytarabine in 57 patients did not show any efficacy in treating HIV-positive subjects with PML, whereas another study reported positive clinical efficacy results in 7/19 (36\%) of the patients [3]. We used cidofovir and cytarabine based on a successful report [8]. Mirtazapine was added to her therapy because in vitro studies have suggested a role for the 5-HT2a receptors acting as the receptor for JCV in glial cells in the brain [3]. We added mefloquine, a widely used antimalarial drug that showed some efficacy in inhibiting the JCV infection be- 
Ghaderi Berntsson et al.: Progressive Multifocal Leukoencephalopathy and Systemic Lupus Erythematosus: Focus on Etiology

cause of in vitro data as well as clinical improvement in patients with PML [3]. A clinical case study with a combination of the antiviral agent CMX001, an oral formulation of cidofovir and recombinant human interleukin-7 (CYT107), an inducer of CD4+ and CD8+ T cells, reported successful treatment in PML [3]. Unfortunately, these investigational drugs were not available at the time our patient required treatment.

Current guidelines on the treatment of SLE suggest that antimalarials may be of benefit in SLE patients without major organ manifestations [15]. We were unable to understand why the patient was not on hydroxychloroquine, which may have provided symptomatic control for the arthralgia and spared her from IST. JC polyomavirus screening for PML risk stratification in patients under IST should perhaps be extended to SLE patients with longstanding lymphopenia in order to prevent viral reactivation in this setting. The present case study highlights several aspects in SLE patient management. JCV infection should be considered at the onset of neuropsychiatric symptoms, total lymphocyte and lymphocyte subset counts should be performed at regular intervals and, most importantly, IST leading to lymphopenia should be avoided for as long as possible.

\section{Statement of Ethics}

The patient's partner has agreed to the publication by written consent. Despite several attempts, her parents could not be contacted, and she had no siblings.

\section{Disclosure Statement}

The authors declare that there are no conflicts of interest regarding the publication of this paper.

\section{References}

1 Astrom KE, Mancall EL, Richardson EP Jr: Progressive multifocal leuko-encephalopathy; a hitherto unrecognized complication of chronic lymphatic leukaemia and Hodgkin's disease. Brain 1958;81:93-111.

-2 Haider S, Nafziger D, Gutierrez J A, Brar I, Mateo N, Fogle J: Progressive multifocal leukoencephalopathy and idiopathic CD4+lymphocytopenia: a case report and review of reported cases. Clinl Infect Dis 2000;31:E20E22.

3 De Gascun CF, Carr MJ: Human polyomavirus reactivation: disease pathogenesis and treatment approaches. Clin Dev Immunol 2013;2013:373579.

4 Molloy ES, Calabrese LH: Progressive multifocal leukoencephalopathy: a national estimate of frequency in systemic lupus erythematosus and other rheumatic diseases. Arthritis Rheum 2009;60:3761-3765.

5 Tsang-A-Sioje M, Bultink I: Systemic lupus erythematosus: review of synthetic drugs. Expert Opin Pharmacother 2015;16:2793-2806.

-6 Calabrese LH, Molloy ES, Huang D, Ransohoff RM: Progressive multifocal leukoencephalopathy in rheumatic diseases: evolving clinical and pathologic patterns of disease. Arthritis Rheum 2007;56:2116-2128.

7 Brandao M, Damasio J, Marinho A, da Silva AM, Vasconcelos J, Neves E, Almeida I, Farinha F, Vasconcelos C: Systemic lupus erythematosus, progressive multifocal leukoencephalopathy, and T-CD4+ lymphopenia. Clin Rev Allergy Immunol 2012;43:302--307.

8 Blick G, Whiteside M, Griegor P, Hopkins U, Garton T, LaGravinese L: Successful resolution of progressive multifocal leukoencephalopathy after combination therapy with cidofovir and cytosine arabinoside. Clin Infect Dis 1998;26:191-192.

-9 Futrell N, Schultz LR, Millikan C: Central nervous system disease in patients with systemic lupus erythematosus. Neurology 1992;42:1649-1657.

10 Berger JR, Aksamit AJ, Clifford DB, Davis L, Koralnik IJ, Sejvar JJ, Bartt R, Major E O, Nath A: PML diagnostic criteria: consensus statement from the AAN Neuroinfectious Disease Section. Neurology 2013;80:14301438. 
Ghaderi Berntsson et al.: Progressive Multifocal Leukoencephalopathy and Systemic Lupus Erythematosus: Focus on Etiology

11 Brink NS, Miller RF: Clinical presentation, diagnosis and therapy of progressive multifocal leukoencephalopathy. J Infect 1996;32:97-102.

12 Merayo-Chalico J, Gomez-Martin D, Pineirua-Menendez A, Santana-De Anda K, Alcocer-Varela J: Lymphopenia as risk factor for development of severe infections in patients with systemic lupus erythematosus: a case-control study. QJM 2013;106:451-457.

13 Rivero SJ, Diaz-Jouanen E, Alarcon-Segovia D: Lymphopenia in systemic lupus erythematosus. Clinical, diagnostic, and prognostic significance. Arthritis Rheum 1978;21:295-305.

14 Levine AB, Erkan D: Clinical assessment and management of cytopenias in lupus patients. Curr Rheumatol Rep 2011;13:291-299.

-15 Bertsias G, Ioannidis JP, Boletis J, Bombardieri S, Cervera R, Dostal C, Font J, Gilboe IM, Houssiau F, Huizinga $\mathrm{T}$, et al: EULAR recommendations for the management of systemic lupus erythematosus. Report of a Task Force of the EULAR Standing Committee for International Clinical Studies Including Therapeutics. Ann Rheum Dis 2008;67:195-205.

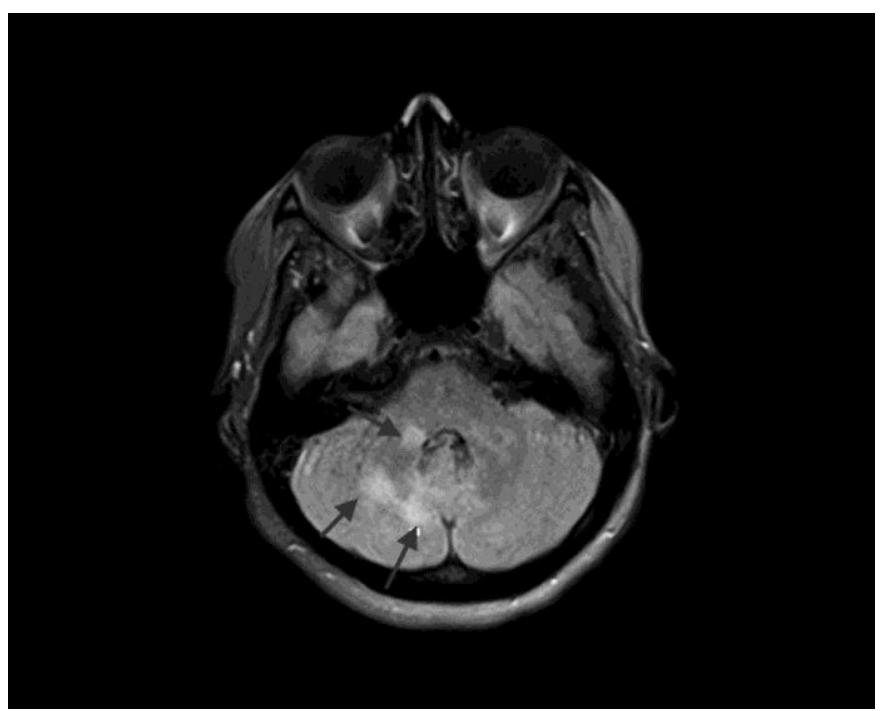

Fig. 1. MRI at the onset of neurological symptoms. Axial T2-weighted fluid-attenuated inversion recovery (FLAIR) shows patchy high-signal intensity PML lesions in the right cerebellar peduncle. 


\section{Case Reports in Neurology}

\begin{tabular}{l|l}
\hline \multicolumn{2}{l}{ Case Rep Neurol 2016;8:59-65 } \\
\hline DOI: 10.1159/000444874 & $\begin{array}{l}\text { (c) 2016 The Author(s). Published by S. Karger AG, Basel } \\
\text { www.karger.com/crn }\end{array}$ \\
\hline
\end{tabular}

Ghaderi Berntsson et al.: Progressive Multifocal Leukoencephalopathy and Systemic Lupus Erythematosus: Focus on Etiology

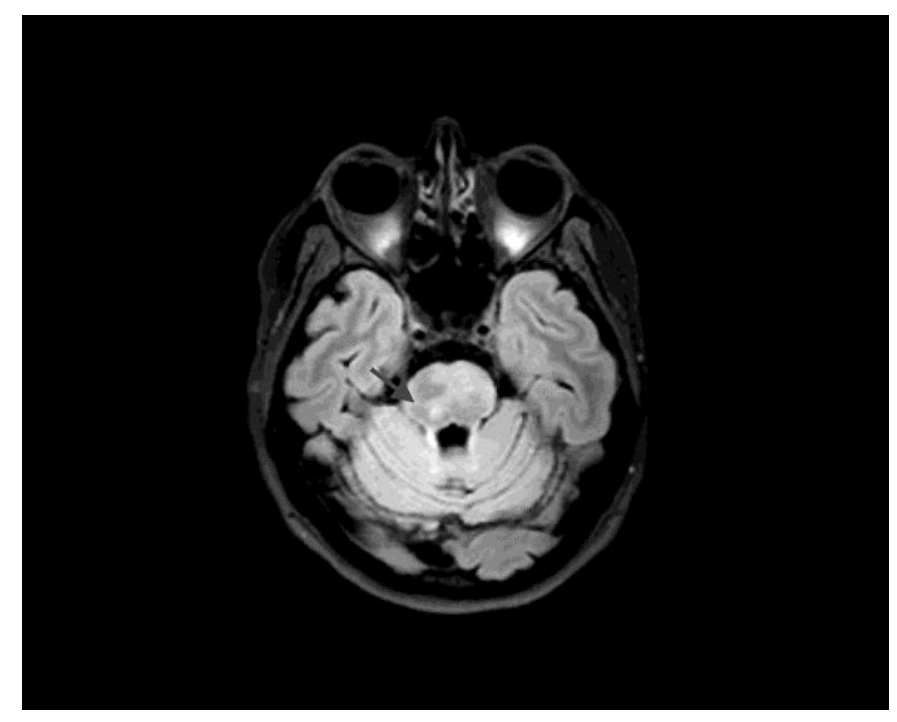

Fig. 2. MRI 5 months later. T2-weighted FLAIR shows a progress of high-signal intensity in the pons.

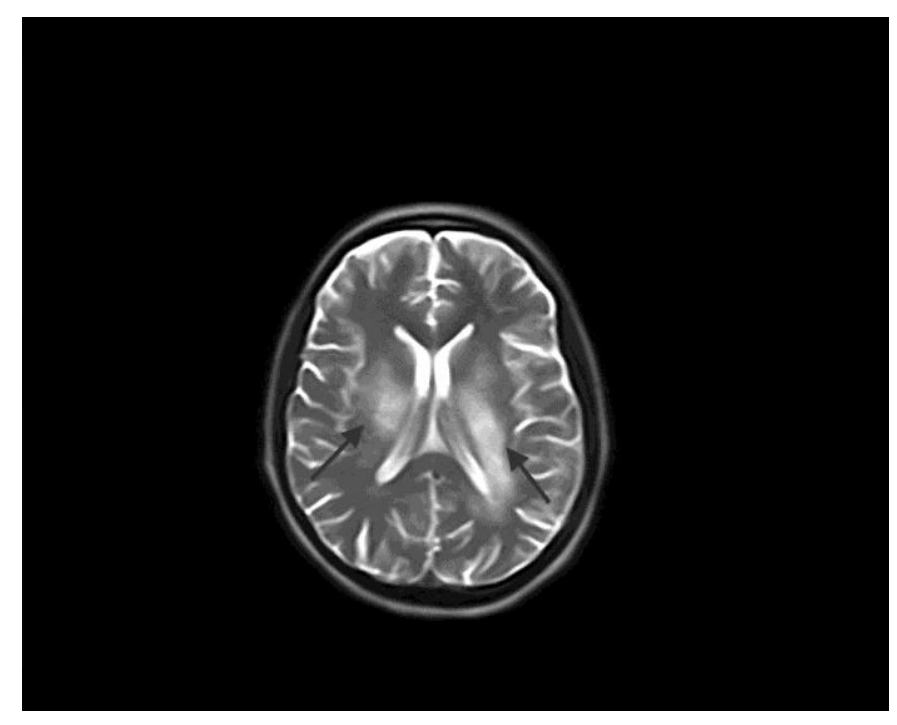

Fig. 3. MRI at the terminal stage of the disease. Axial T2-weighted MRI shows periventricular high-signal intensity. 